\title{
MEDIAL SEPTAL AREA LESIONS DISRUPT $\theta$ RHYTHM AND CHOLINERGIC STAINING IN MEDIAL ENTORHINAL CORTEX AND PRODUCE IMPAIRED RADIAL ARM MAZE BEHAVIOR IN RATS ${ }^{1}$
}

\author{
SUSAN J. MITCHELL, ${ }^{* 2}$ J. N. P. RAWLINS, ${ }^{* 3}$ O. STEWARD, \\ *Department of Psychology, The Johns Hopkins University, Baltimore, Maryland 21218 and $\ddagger$ Department of Neurosurgery, \\ The University of Virginia School of Medicine, Charlottesville, Virginia 22908
}

Received July 23, 1981; Revised October 29, 1981; Accepted October 30, 1981

\begin{abstract}
This study was designed to determine (1) which brain area paces the $\theta$ rhythm in the medial entorhinal cortex (MEC) of rats and (2) the extent to which the behavioral effects of lesions in the medial septal area (MSA), which disrupt the cholinesterase-related pathway to the hippocampal formation, resemble the effects previously reported to result from fimbria-fornix lesions.

MSA lesions abolished or decreased $\theta$ rhythm in dorsal hippocampus (DHPC) and MEC; acetylcholinesterase (AChE) staining was depleted or diminished in all of the hippocampus and entorhinal cortex. Rats with MSA lesions were impaired on acquisition of a radial arm maze task.

Unilateral fimbria lesions left $\theta$ rhythm and AChE staining essentially unaltered in ipsilateral DHPC and MEC but depleted AChE in ipsilateral ventral hippocampus (VHPC) and ventral lateral entorhinal cortex (LEC). A lesion of the dorsal fornix at the level of the hippocampal flexure left ipsilateral DHPC $\theta$ rhythm and $\Lambda \mathrm{ChE}$ stain unaltered while causing a substantial reduction in $\theta$ rhythm and depletion of AChE in ipsilateral MEC. AChE staining was complete in VHPC and LEC.

These results suggest that MSA paces MEC $\theta$ rhythm and that the presumed cholinergic projection which mediates this function travels in the dorsal fornix. The fimbria carries a presumed cholinergic projection to ventral LEC. Rats with MSA lesions can learn a radial arm maze task, unlike rats with fimbria-fornix lesions, but they learn significantly slower than normal rats.
\end{abstract}

The hippocampal $\theta$ rhythm observed in lower mammals has two separate generators. One is located in Ramón y Cajal's regio superior and the other is in the dentate gyrus (Bland et al., 1975; Bland and Whishaw, 1976; Green et al., 1960; Green and Petsche, 1961; Winson, $1974,1976 \mathrm{a}, \mathrm{b})$. Recently, at least one and possibly two generators of $\theta$ activity have been found in the medial entorhinal cortex (MEC) (Mitchell and Ranck, 1980). In rats, when $\theta$ rhythm is present in one hippocampal generator, it is nearly always present in the other (Bland and Whishaw, 1976; Green and Rawlins, 1979) and in the MEC (Mitchell and Ranck, 1980). The amplitude modulation of the $\theta$ rhythm is similar in regio superior and

\footnotetext{
1 This work was supported by National Research Service Award 5 F32 MH07702 to S. J. M., Fogarty International Center Fellowship 1 F05 TW02804 to J. N. P. R., and United States Public Health Service Grant MH24213 to D. S. O., all of which we gratefully acknowledge.

${ }^{2}$ To whom correspondence should be addressed at her present address: Department of Neurology, Baltimore City Hospital, B Building, Room 122, 4940 Eastern Avenue, Baltimore, MD 21224.

${ }^{3}$ Present address: Department of Experimental Psychology, South Parks Road, Oxford OXI 3UD, England.
}

layer III of the MEC and in the dentate gyrus and layer II of the MEC. The frequency modulation of all four signals is identical (Mitchell and Ranck, 1980). These observations suggest that there is a pacemaker for the $\theta$ rhythm outside of the hippocampal formation (here used to mean Ammon's horn and the dentate gyrus, the subicular cortices, and entorhinal cortex).

In the hippocampus (Ammon's horn and the dentate gyrus), the maintenance of $\theta$ rhythm is thought to depend upon the rhythmical activity of neurons located in the medial septal nucleus and the nucleus of the diagonal band (Apostol and Creutzfeldt, 1974; Gogolák et al., 1967, 1968; McLennan and Miller, 1974, 1976; Petsche et al., 1962, 1965; Ranck, 1976; Wilson et al., 1976). These nuclei are considered to constitute the "medial septal area" (MSA).

MSA has a major projection to all of the cell fields of the hippocampus (HPC) via fibers running in the fimbria and fornix (Raisman, 1966; Meibach and Siegel, 1977; Swanson and Cowan, 1977). Disrupting this projection, whether by making lesions in the medial septal area or in the fornix and fimbria, can abolish hippocampal $\theta$ activity; $\theta$ in the septal pole of the hippocampus appears 
to depend upon fibers running in the dorsomedial portion of the fornix, while $\theta$ in the temporal poles depends on fibers running in the fimbria (Rawlins et al., 1979).

The areas in which $\theta$ activity is disrupted by MSA lesions, or by selective lesions of the fibers in different parts of the fornix or fimbria bundle, correspond well to regional depletions of acetylcholinesterase (AChE) staining in the hippocampus produced by the same lesions (Mellgren and Srebro, 1973; Rawlins et al., 1979). It thus seems that hippocampal $\theta$ rhythm is controlled by a topographically organized, and possibly cholinergic, input from the medial septal area.

The MS $\Lambda$ also projects to all of the entorhinal cortex (EC) diffusely across all cortical layers (Meibach and Siegel, 1977; Segal, 1977; Siegel and Tassoni, 1971; Swanson and Cowan, 1977). The route taken by the fibers of this pathway has been identified for only the most ventral portion of EC (Meibach and Siegel, 1977). Acetylcholinesterase activity is present in EC (Storm-Mathisen and Blackstad, 1964) and is depleted following lesions of the MSA (Mellgren and Srebro, 1973). It is thus possible that the MSA also paces EC $\theta$ rhythm via a projection resembling that from MSA to HPC.

The entorhinal cortex contributes a massive and highly organized input to the hippocampus via the perforant path (Ramón y Cajal, 1955). If MSA paces EC $\theta$ rhythm, then subcortical MSA activity which produced a potentially modulatory action on hippocampal cells (Artemenko, 1972; Fujita and Sato, 1964; Fox, 1980) also may modulate EC cells. Among the modulated EC cells may be those cells which project via the perforant path to the hippocampus. If this is true, our view of how the hippocampal formation functions and is modulated must be altered somewhat.

Behaviorally, the hippocampus has been implicated as a brain structure important for the normal processing of memory (Olton et al., 1979), particularly working memory (Honig, 1978; Olton and Samuelson, 1976), a relatively short term, trial-specific memory. For example, bilateral lesions of the fimbria-fornix or EC produced a severe impairment in the ability of rats to choose accurately in a radial arm maze task which requires working memory (Olton et al., 1978, 1979). A unilateral lesion of the fimbria-fornix combined with dorsal and ventral HPC commissure cuts and a lesion of contralateral EC produce the same deficit. Thus, destruction of the MSA or the fiber bundles which project from MSA to the hippocampal formation might be expected to produce a behavioral impairment in the same task.

The first objective of the present study was to determine whether MEC $\theta$ rhythm is paced by a direct input from MSA and, if so, how the fibers from MSA travel to MEC. The method used was to make lesions of MSA and then subsequently of the fimbria and dorsal fornix. Following these lesions, changes in slow wave activity and AChE staining in HPC and EC were assessed.

The second objective of this study was to measure the choice accuracy of rats with selective lesions in the working memory radial arm maze task. Rats with MSA lesions and unilateral fimbria lesions were tested on the radial arm maze and the correlation between the observed behavioral impairments and the observed changes in $\theta$ rhythm and AChE staining was determined.

\section{Materials and Methods}

\section{General procedure}

Adult male albino rats were used in this study. There were four test groups: MSA lesion (seven rats), unilateral fimbria lesion (six rats), dorsal fornix lesion (seven rats), and operated control (five rats). The experimental procedure for each of the lesion groups was the same. All of the rats had recording electrodes implanted and the MSA group and dorsal fornix group had lesion electrodes implanted as well. After the rats recovered from surgery, slow waves were obtained from all recording electrodes. Lesions then were made in the rats, and slow waves were obtained again from all recording electrodes at 1 to 8 days following the lesion and periodically for as long as $6^{1 / 2}$ months. During this time, the rats were trained to run on a radial arm maze and tested on it for 30 sessions. The operated control group had recording electrodes and MSA lesion electrodes implanted. Slow wave recordings were made as before and the animals were trained to run and tested for 30 sessions on the radial arm maze. For all groups, when the behavioral experiment was completed, the animals were perfused and their brains were processed for histological examination.

\section{Surgery}

Following an injection of $0.3 \mathrm{ml}$ of atropine methyl nitrate (Sigma Chemical Corp., $0.5 \mathrm{mg} / \mathrm{ml}$ ), the animals were anesthetized with Chloropent (Fort Dodge Laboratories, $2.5 \mathrm{ml} / \mathrm{kg}$ ), and $0.1 \mathrm{ml}$ of Bicillin (Wyeth, 300,000 units $/ \mathrm{ml}$ ) was administered intramuscularly to each hind leg. The rat's head was level in a stereotaxic device, bregma and lambda in the same horizontal plane, for placement of electrodes. A pair of recording electrodes (twisted 150- $\mu \mathrm{m}$ SS No. 316-L, H-ML insulated wire, California Fine Wire, Grover City, CA) was lowered to each recording site. For electrodes in dorsal hippocampus (DHPC), tip separation was 0.5 to $1.0 \mathrm{~mm}$ vertically and coordinates for the recording site were $4.2 \mathrm{~mm}$ posterior and $2.7 \mathrm{~mm}$ lateral to bregma with the deeper tip $4.0 \mathrm{~mm}$ from the surface of the skull. MEC electrodes had tips separated vertically by 1.0 to $1.5 \mathrm{~mm}$, were slanted anteriorly in the sagittal plane to form an angle of $40^{\circ}$ with the skull surface, and were lowered to a point $5.0 \mathrm{~mm}$ dorsal, $5.2 \mathrm{~mm}$ lateral, and $0.8 \mathrm{~mm}$ anterior to earbar zero.

For rats in the MSA group, a blunt cut lesion electrode made of $250-\mu \mathrm{m}$-diameter SS Diamel insulated wire was placed $1.0 \mathrm{~mm}$ anterior to bregma and lowered through the sagittal sinus to a depth of $5.0 \mathrm{~mm}$ from the surface of the sinus. For rats in the dorsal fornix group, lesion electrodes were made of the same material, but the tips were cut at approximately $45^{\circ}$ and the insulation was scraped away $0.5 \mathrm{~mm}$ from the tip. They were placed bilaterally at $5.6 \mathrm{~mm}$ posterior and $1.5 \mathrm{~mm}$ lateral to bregma and $3.2 \mathrm{~mm}$ from the surface of the skull. For rats in the fimbria group, an area of skull large enough to allow subsequent access to the fimbria was left free of acrylic, and the bone was covered with a film of Vaseline.

In all animals, small self-tapping screws placed in the frontal and interparietal bones served to anchor the implant to the rat's skull. The left frontal bone screw served as the indifferent electrode for all recordings; one 
of the interparietal screws served to ground the rat. The electrodes were secured to the screws and skull with dental acrylic. A 2:1 mixture of sulfadiazine and sulfanilamide (Sigma Chemical Corp.) was sprinkled liberally at the edges of the wound.

\section{Recording}

All slow waves were recorded differentially between each individual electrode and the frontal bone screw. The signals were led through source follower field effect transistors in a head stage firmly connected to the rat's implant. Slow waves were filtered 1 to $100 \mathrm{~Hz}$, monitored on an oscilloscope, and recorded by a Narco Physiograph (DMP-4B).

All slow wave records were obtained while the rats walked in a treadmill which ran at a constant speed (Mitchell and Ranck, 1980).

\section{Lesions}

Rats were anesthetized with ether and MSA lesions were made by passing $1 \mathrm{~mA}$ of DC current for $15 \mathrm{sec}$ using the lesion electrode as an anode. Dorsal fornix lesions were made by passing $10 \mathrm{~mA}$ of radiofrequency current, from a Grass LM-4 Lesion Maker, for 15 sec. Return current was carried by a rectal electrode. The fimbria was removed unilaterally, by suction, using the procedure of Rawlins et al. (1979). In four rats which did not have implanted electrodes, unilateral suction lesions were made of the posterior dorsal fornix by removing fibers at $5.6 \mathrm{~mm}$ posterior to bregma and for several millimeters posterior to that point.

\section{Behavioral testing}

Animals were trained to run on a radial arm maze which has been described in detail elsewhere (Becker et al., 1980). It was elevated above the floor and had eight arms radiating from a central platform. At the end of each arm was a hole which served as a food cup. Around the central platform was a Plexiglas wall with a guillotine door at the entrance to each arm.

At the beginning of each test session, one pellet of food (P. J. Noyes Co., $190 \mathrm{mg}$ ) was placed at the end of each arm. A rat was placed in the center of the maze with all of the guillotine doors closed. All of the doors were raised, allowing the rat to choose an arm. When the rat returned to the central platform, the guillotine doors were lowered, confining him there for approximately $5 \mathrm{sec}$. All of the doors were raised again, and this procedure continued until the rat had been to all eight arms, had not made a choice for $2 \mathrm{~min}$, or had spent a total of $10 \mathrm{~min}$ in that test session. Choosing a baited arm was scored as a correct response; revisiting a previously chosen arm within the first eight choices was scored as an error.

Rats were given one test session per day, 5 days per week during the period of behavioral testing. Criterion performance was seven correct responses in the first eight choices of each test session for five consecutive test sessions.

\section{Histology}

Animals were anesthetized deeply, and 5 to $7 \mu \mathrm{A}$ of current were passed for 7 to $10 \mathrm{sec}$ through each recording electrode with the electrode positive and return current was carried by an alligator clip on the rat's skin at the edge of the implant. They were immediately perfused transcardially with physiological saline followed by $10 \%$ formalin in physiological saline. The brains were blocked by a cut in the coronal plane just caudal to the site of entry of the HPC recording electrodes. Each block was embedded in egg yolk, fixed, frozen, and cut in $40-\mu \mathrm{m}$ sections in the coronal plane for the rostral block and in the horizontal plane for the caudal block. Every fifth section was stained for AChE (Naik, 1963). A few sections of brain from rats in several unilateral fimbria and dorsal fornix groups were stained for fibers by the Nauta-Gygax method (Humason, 1979). The remaining sections were immersed in $2 \%$ ferrocyanide in physiological saline for $36 \mathrm{hr}$ and then rinsed and stained for Nissl substance with cresyl violet.

Recording sites were identified by location of the electrode tip and the Prussian blue reaction product.

\section{Data analysis}

$\theta$ rhythm. The amplitude of the $\theta$ rhythm was assessed by measuring the peak-to-peak amplitude of 20 consecutive waves and calculating the mean value. For each electrode site, the extent of the $\theta$ rhythm loss following a lesion was expressed as a percentage of the amplitude of the $\theta$ rhythm prior to the lesion. For this to be an accurate measure, the electrode tips must not move with respect to the $\theta$ generators. Assessment for movement was made by comparing the amplitude patterns and phase relations between the two electrodes of a pair before and after the lesion (Mitchell and Ranck, 1980; Winson, 1974). With the exception of a few pairs of electrodes in the unilateral fimbria group (see "Results") and a few pairs in the dorsal fornix group, there was no evidence of electrode movement.

The numerical values for $\theta$ rhythm loss determined for each electrode site were used to compare the loss in HPC and MEC sites within a rat by calculating a correlation coefficient.

A measure of overall $\theta$ rhythm loss for each rat was obtained by calculating the mean $\theta$ rhythm loss at all electrode siles from which $\theta$ rhythm had been recorded before the lesion. These values were used to rank order the animals for overall $\theta$ rhythm loss. All rank-ordered parameters were evaluated statistically using Spearman's rank correlation statistics.

$A C h E$. AChE depletion was evaluated by two observers who examined DHPC, ventral HPC (VHPC), dorsal MEC, and ventral EC bilaterally in each rat. Each area was ranked on a five-point scale which ranged from complete loss to complete retention. The mean of these values was used to rank order the animals for overall AChE loss.

Behavioral impairment. The number of test sessions to reach criterion was calculated for each rat and used to evaluate differences between the groups. To rank order the animals within a group, a more precise measure of performance was used for overall behavioral impairment. For each animal, two measures were made, the number of errors to reach criterion and the number of errors during the last five sessions. This rank order of rats thus 


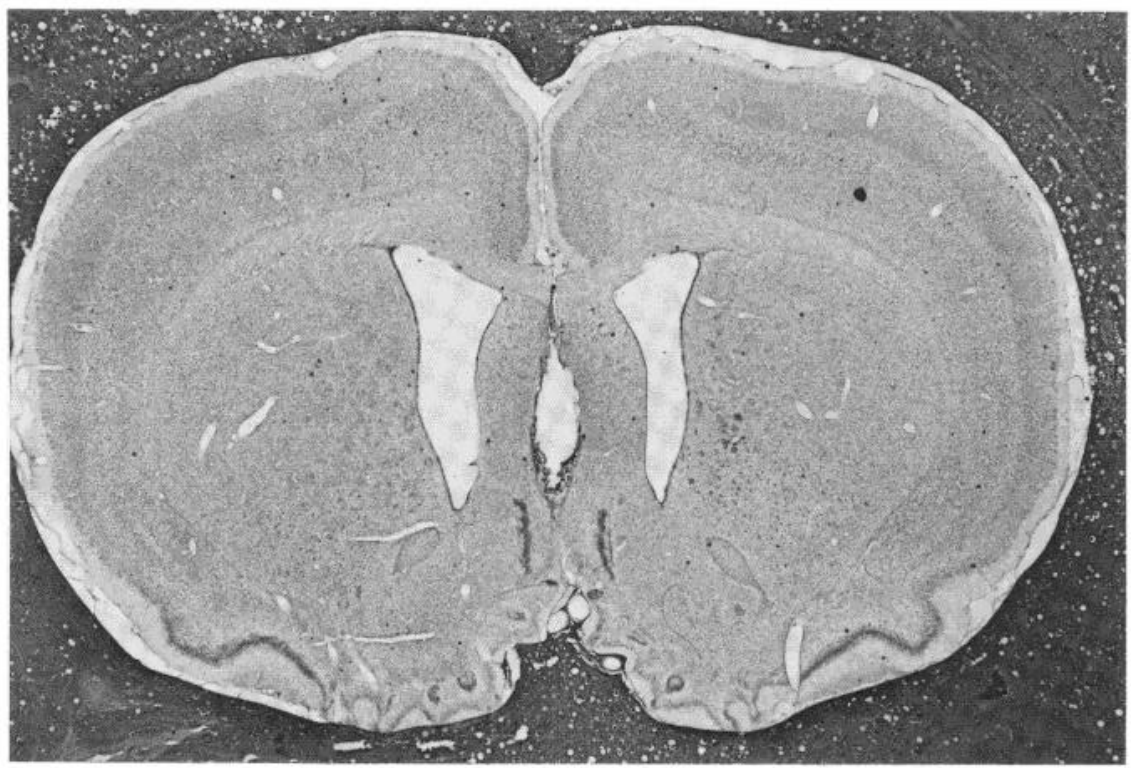

Figure 1. A coronal section at the level of the septal nuclei showing the MSA lesion at its maximum extent. Cresyl violet stain was used; this section is from rat E7.

reflects the choice accuracy of the rats during the learning period, the length of time required to learn, and the choice accuracy during asymptotic performance.

\section{Results}

\section{The MSA lesion}

MSA lesions were produced in seven rats. The lesion typically produced damage throughout the rostrocaudal extent of the MSA, sparing cells of the lateral septal area, and was comparable to previously reported lesions (Rawlins et al., 1979). Figure 1 illustrates a typical lesion at its maximum dorsoventral extent.

Loss of $\theta$ rhythm. Slow waves were recorded from all electrode sites (right DHPC and right MEC in all animals, left MEC in five rats) 1 day after the lesion and at weekly intervals throughout the period of behavioral testing. On the 1st post-lesion day, the loss of $\theta$ rhythm in the right DHPC was complete in three animals and incomplete in the remaining four. The range was 100 to $33 \%$, with a median of $58 \%$ for the whole group. The right MEC suffered losses of 100 to $11 \%$, with a median of $60 \%$, and the left MEC had losses of 100 to $46 \%$, with a median of $68 \%$. Figure 2 shows $\theta$ rhythm recorded from one rat before and after this lesion.

Since the MSA projections to hippocampal formations are not crossed and the lesions were not always perfectly centered, one must compare HPC and MEC on one side of the brain to obtain a meaningful comparison of changes between these structures within one rat. On each rat, the extent of $\theta$ loss in right DHPC was correlated closely with the amount of loss in right MEC $(r=0.98)$.

No recovery of $\theta$ rhythm was observed either at 1 week (range of loss for right HPC, 100 to $30 \%$; median, $51 \%$ : for right MEC, 100 to 6\%; median, 58\%: for left MEC, 100 to $41 \%$; median, $50 \%$ ) or in the final record (range of loss for right HPC, 100 to $33 \%$; median, $46 \%$ : for right MEC, 100 to $25 \%$; median, $47 \%$ : for left MEC, 100 to $39 \%$; median,
$42 \%)$. The final record was made over a period which varied for each rat, ranging from 46 to 104 days.

Loss of AChE. Following the MSA lesions, AChE staining was decreased in dorsal and ventral HPC and medial and lateral EC. The extent of change varied from a very marked loss to near complete retention. Losses in the HPC were similar to EC losses in each rat as illustrated in Figure 3. The overall $\theta$ rhythm loss was correlated significantly with the AChE loss (Spearman's $r_{s}=$ $0.82 ; N=7 ; p<0.05$, one-tailed test).

Behavioral impairment. Rats were trained on the eight-arm maze beginning 14 to 64 days after the MSA lesion had been made. Six of the seven animals reached criterion performance within 30 test sessions. These animals learned the task significantly slower than operated controls as illustrated in Figure 4. The overall $\theta$ rhythm loss was highly, but not significantly, correlated with overall behavioral impairment (Spearman's $r_{s}=0.64 ; N$ $=7$; not significant, one-tailed test), and the overall
BEFORE LESION

RDHPC

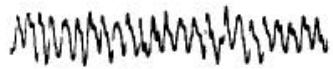

RMEC

LMEC
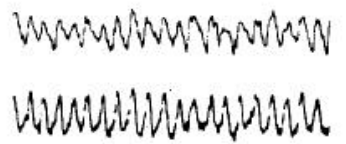

AFTER LESION
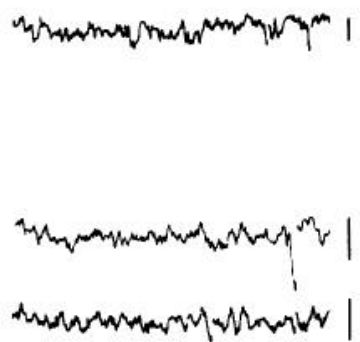

Figure 2. Slow waves from the right DHPC $(R D H P C)$ and the right and left MEC (RMEC and $L M E C$ ) before and 7 weeks after the MSA lesion. The EC signals were recorded simultaneously in this and all subsequent slow wave records. Calibration bars, $500 \mu \mathrm{V}$ and $1 \mathrm{sec}$. Data are from rat E7. 

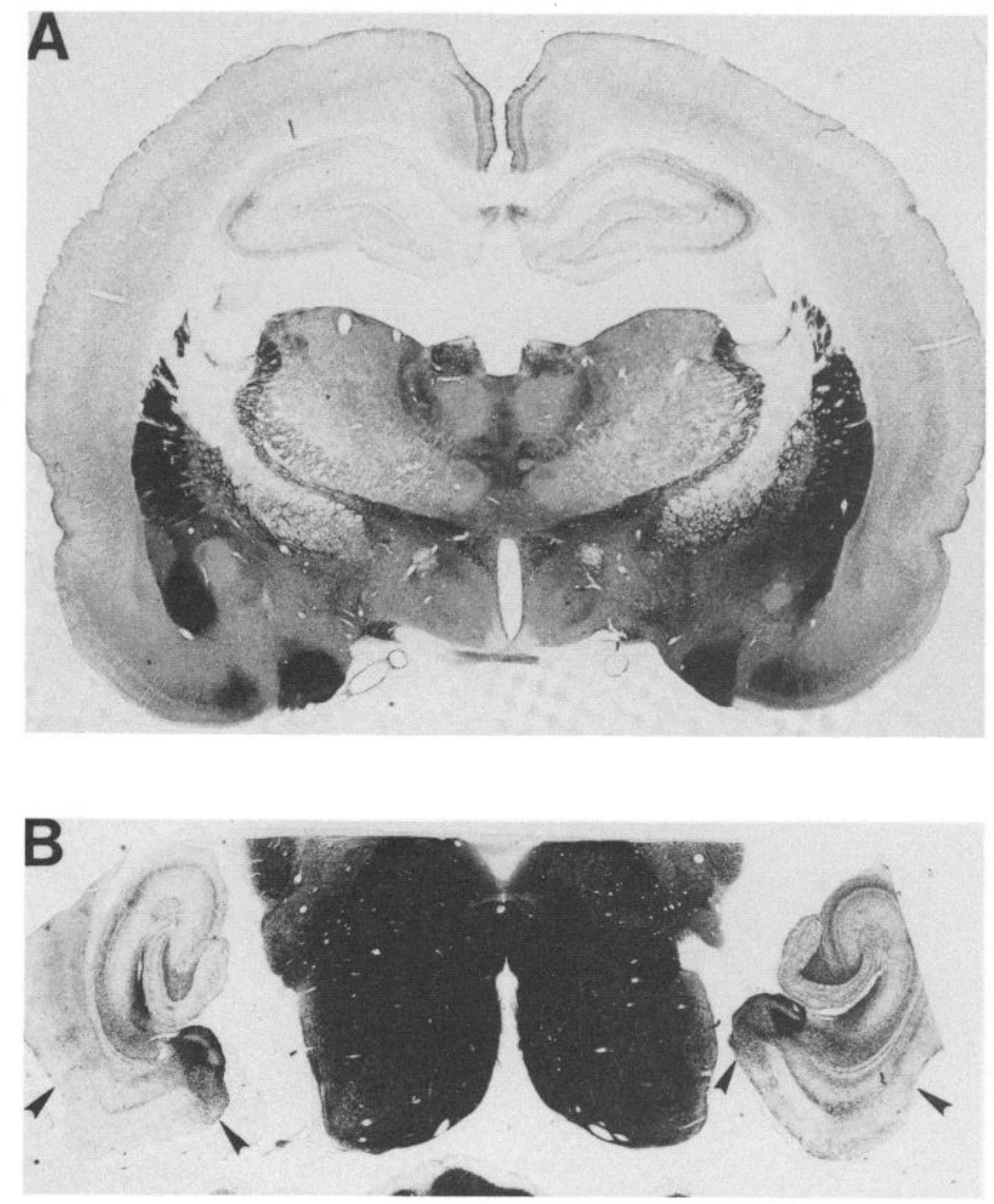

Figure 3. AChE staining pattern in the brain of a rat with an MSA lesion. $A, \mathrm{~A}$ coronal section showing DHPC; $B$, a horizontal section showing EC. Arrowheads mark the medial and lateral borders of EC. Data are from rat E7. For comparison to the pattern seen in a normal rat brain, see Figure $7 A$ for DHPC and Figure $7 B$ for EC.

AChE loss was correlated identically with overall behavioral impairment.

Since testing was begun at various intervals following the lesion, it is possible that some behavioral recovery might occur during the interval. This possibility was tested by calculating a Spearman's rank correlation between the time elapsed between lesion and testing and the number of test sessions to criterion. The correlation did not approach significance (Spearman's $r_{s}=0.15 ; N$ $=7$; not significant, one-tailed test).

\section{Results of the fimbria lesion}

Unilateral fimbria lesions were produced in six rats. The lesion technique used typically produced a major loss of fimbria fibers. There was minimal, if any, damage to the HPC and occasionally some unilateral damage to nearby subcortical structures, including the dorsolateral septum. Figure 5 illustrates one such lesion. A small portion of isocortex directly above the anterior aspect of the fimbria was ablated.

Loss of $\theta$ rhythm. Slow waves were recorded from the DHPC and the MEC on the side ipsilateral to the lesion in all rats and, in addition, from the contralateral MEC in four animals. Figure 6 shows $\theta$ rhythm from one rat before and after the lesion. The first post-lesion recording was made as soon as the animals were judged fit to walk in the treadmill ( 2 to 8 days after the lesion). In the ipsilateral DHPC, the extent of loss was from 40 to $2 \%$, with a median of $15 \%$, and in the ipsilateral MEC, the loss was from 49 to $-22 \%$ (an increase of $22 \%$ ), with a median of $18 \%$. The contralateral MEC sustained little or no loss of $\theta$ rhythm (range, 4 to $-7 \%$; median, $-2.5 \%$ ). The animal which had the maximum loss had a lesion which clearly produced damage to fibers leaving the MSA that are destined for DHPC.

In one rat, the mean amplitude of the $\theta$ rhythm on the 
right MEC electrodes increased by $22 \%$, with one electrode recording a slightly lower amplitude signal and the other recording a much higher amplitude signal than before. This recording pattern suggests that the electrodes' position with respect to the generator site had shifted from its original location. A similar pattern of changes was observed in the final recording from four additional pairs of electrodes, all located in the DHPC ipsilateral to the lesion. In each case, the phase relation between the recordings from the two electrodes also changed from that seen in the initial recording session; similar phase changes were not observed in our other recordings. These changes in the recordings are the appropriate, expected changes which occur as the electrodes move with respect to the generators in these areas

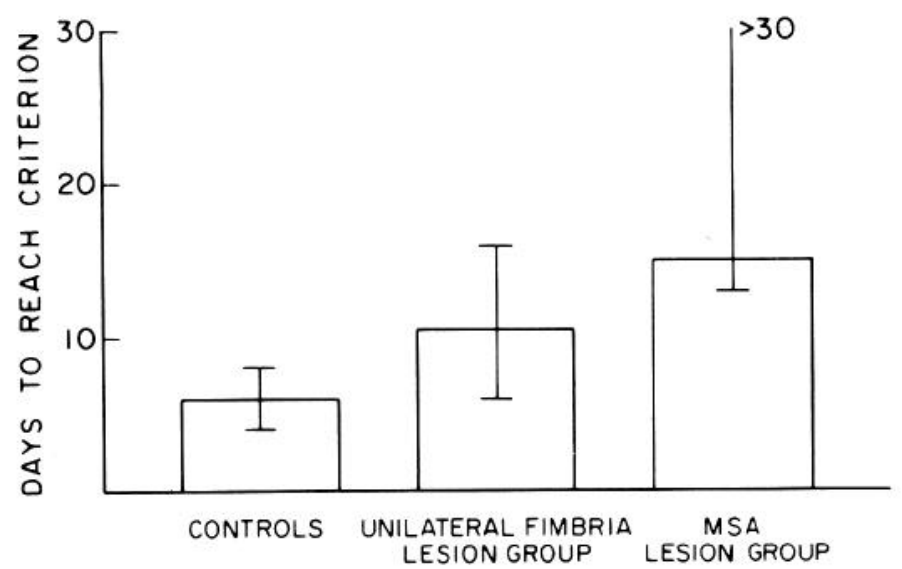

Figure 4. Median number of days and the range for each group before the 1st day of criterion performance. Each of these results differs significantly from the others. Using Mann-Whitney $U$ tests, comparing operated controls to the MSA group, $U$ $=0 ; p=0.002$; comparing operated controls to the unilateral fimbria group, $U=2.5 ; p<0.03$; comparing the MSA group to the unilateral fimbria group, $U=4 ; p=0.014$.
(Mitchell and Ranck, 1980; Winson, 1974). This movement of brain tissue with respect to the electrodes precludes comparison of any of these recordings with earlier ones. However, in the animals with stable MEC electrodes, no recovery of the MEC $\theta$ rhythm was observed (range of loss for ipsilateral MEC, 32 to $0 \%$; median, 21\%, five rats; range of loss for contralateral MEC, 18 to $-9 \%$; median, $3 \%$, three rats).

In summary, there were only slight losses of $\theta$ rhythm in both DHPC and MEC ipsilateral to the lesion in rats with lesions confined to fimbria. The lesion method produced damage which resulted in tissue moving with respect to electrodes frequently in ipsilateral DHPC and once in ipsilateral MEC. Since the range of $\theta$ rhythm loss in these animals was small, it was not possible to rank them and do correlation analyses.

Loss of AChE. The five rats with lesions confined to the fimbria sustained very small or no losses of AChE in DHPC and in MEC. The ventral (or temporal) HPC, however, lost most of its $\mathrm{AChE}$, and the ventral portion
BEFORE LESION

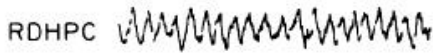

RMEC

LMEC

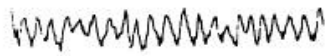

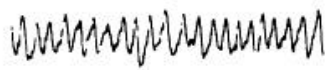

AFTER LESION

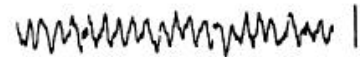

Figure 6. Slow waves from the right DHPC $(R D H P C)$ and the right and left MEC (RMEC and $L M E C$ ) before and 10 days after a right unilateral fimbria lesion. Calibration bars, $500 \mu \mathrm{V}$ and $1 \mathrm{sec}$. Data are from rat E10.

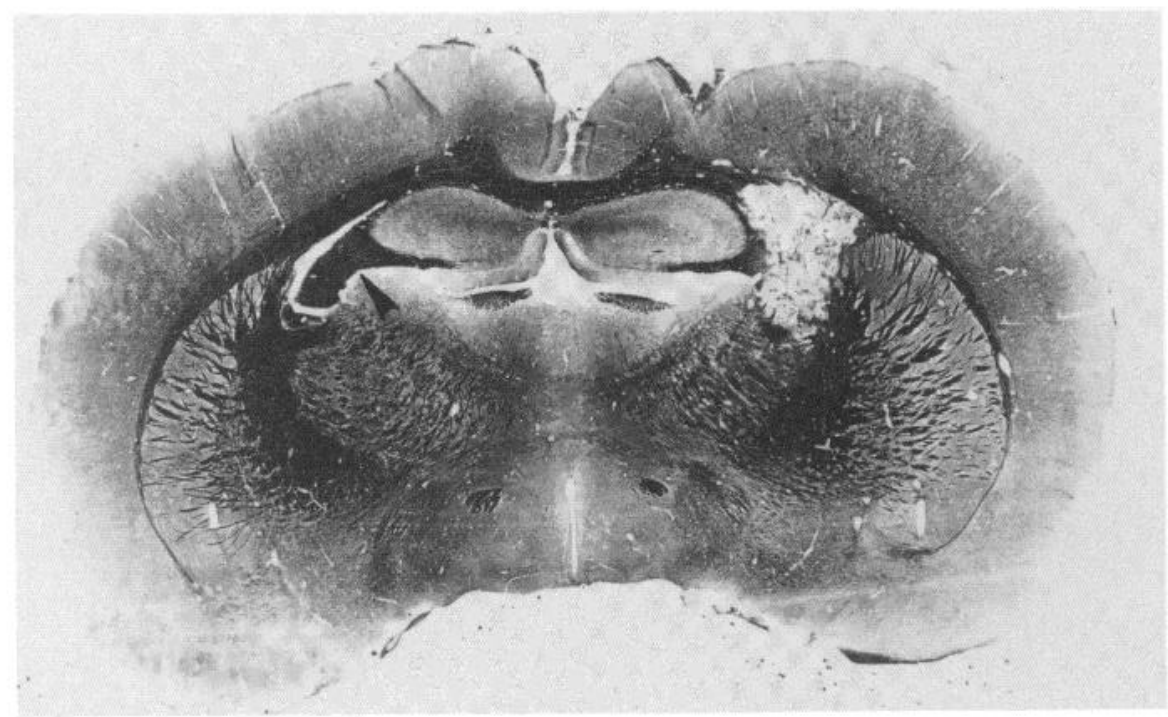

Figure 5. A coronal section at the level of the septal pole of the HPC showing the intact fimbria (see arrowhead) on the left and the absence of fimbria fibers on the right. Nauta-Gygax stain was used; this section is from rat E14. 
of the lateral entorhinal cortex (LEC) lost moderate to large amounts of the stain as illustrated in Figure 7.

Behavioral impairment. The rats were trained to run on the eight-arm radial maze after their lesions had been made and they learned the task significantly faster than rats with MSA lesions but significantly slower than operated controls (Fig. 4).

\section{Results of the dorsal fornix lesion}

All of the dorsal fornix lesions produced by radiofrequency current in rats with implanted electrodes were incomplete. The placement of individual lesions spared fibers dorsally, ventrally, or at the medial or lateral edges at the site of the lesion.

Loss of $\theta$ rhythm. Slow waves were recorded bilaterally from HPC and EC 1 day after the lesion and at weekly intervals for 2 weeks to 1 month. One rat received only a unilateral right side lesion. On the 1st day following this lesion, the right MEC $\theta$ rhythm loss was $47 \%$ and the left MEC loss was $12 \%$, with no loss in HPC on either side. These data are illustrated in Figure 8. All remaining animals received simultaneous bilateral lesions. Two rats
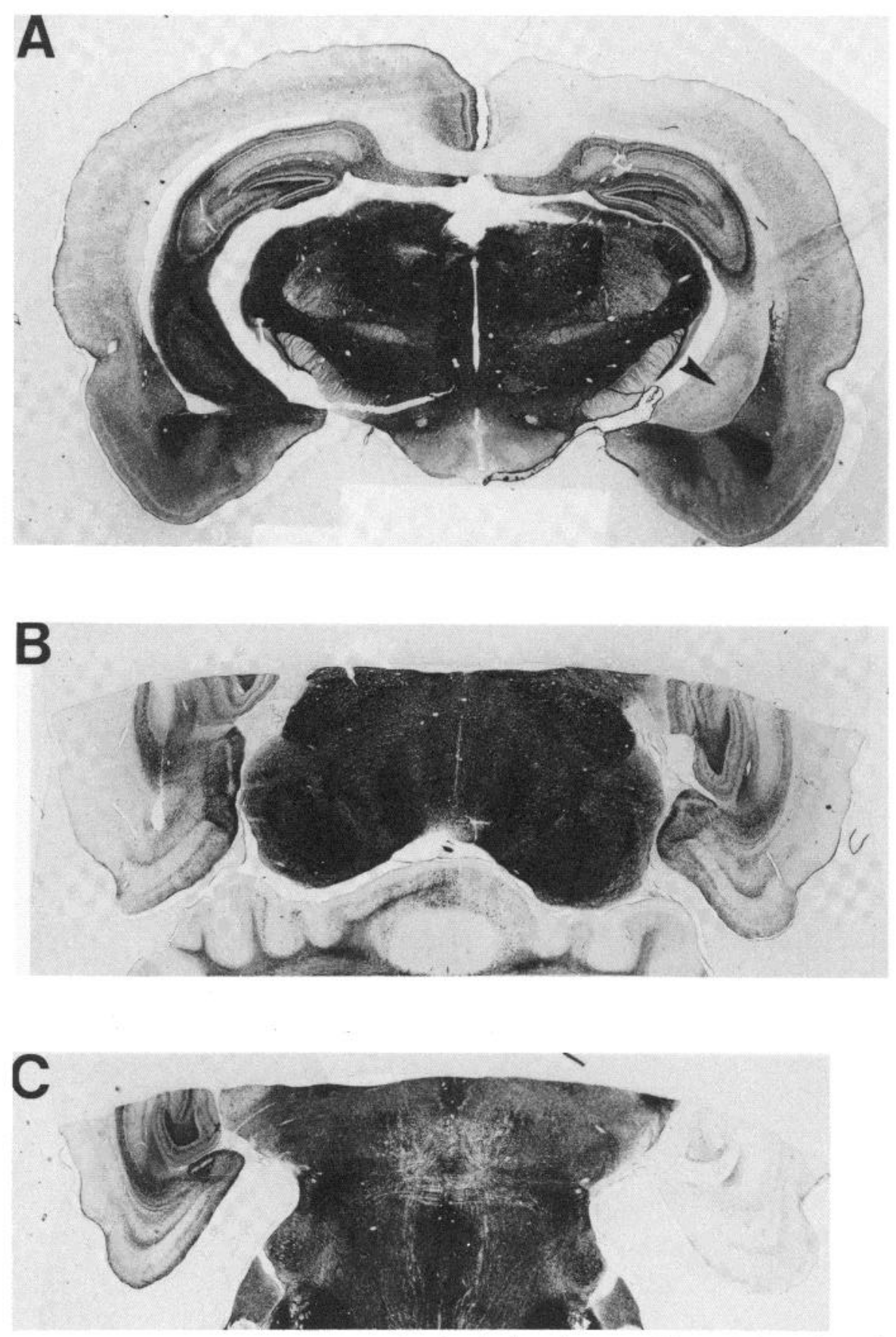

Figure 7. AChE staining pattern in the brain of a rat with a right unilateral fimbria lesion. $A$, A coronal section showing both the dorsal and ventral hippocampus. Note the absence of AChE stain in the right VHPC (see arrowhead). B, A horizontal section showing EC; $C$, a more ventral horizontal section showing LEC. Note the depletion of AChE stain on the right side. These sections are from rat E10. 
sustained approximately equivalent losses in DHPC and MEC. The range for HPC was 74 to $47 \%$, with a median of $64 \%$; the range for $\mathrm{MEC}$ was 48 to $29 \%$, with a median of $46 \%$. The remaining two rats had substantial losses in DHPC (range, 79 to $40 \%$; median, $69 \%$ ) and only slight losses in MEC (range, 21 to $4 \%$; median, 18\%).

Some recovery of the $\theta$ rhythm was observed at 14 days following the lesion. In the rat with the unilateral lesion and one rat with approximately equivalent DHPC and MEC loss, there was evidence for electrode movement in DHPC; in the remaining three rats, DHPC $\theta$ loss ranged from 60 to $27 \%$, with a median of $45 \%$.

The MEC electrodes also moved in the rat with the unilateral lesion. For the remaining four rats, the MEC $\theta$ rhythm recovered somewhat during a period of 14 to 29 days; the range for the animals with the original loss approximately equivalent to DHPC loss was 27 to $-1 \%$, with a median of $14 \%$; the range for the animals with only slight original losses was 18 to $-6 \%$, with a median of $9 \%$.

Loss of AChE. Histological assessment of these rats was made approximately 6 months following the lesions. The AChE staining was found to be extensive or complete in DHPC and MEC in all animals.

Four additional rats which received unilateral suction lesions of dorsal fornix were assessed for $\mathrm{AChE}$ staining at 7 days after the lesion. They showed a marked loss of $\mathrm{AChE}$ in MEC ipsilateral to the lesion and complete retention in DHPC on both sides and in contralateral MEC. These results are illustrated in Figure 9. LEC also showed complete retention bilaterally.

\section{Discussion}

The first objective of the present study was to determine whether MEC $\theta$ rhythm is paced by a direct input from MSA. Lesions of MSA were made and changes in slow wave activity and $\mathrm{AChE}$ staining in $\mathrm{HPC}$ and $\mathrm{EC}$ were assessed. The expected $\theta$ rhythm loss in DHPC was matched by a similar loss in MEC and the decrease in $\theta$ rhythm co-varied with depletion of AChE. These results ruled out the possibilities that MEC $\theta$ rhythm was endogenous or was placed by a central nervous system structure outside of the hippocampal formation. Two possibilities remained: either the medial septal area paced MEC $\theta$ rhythm monosynaptically (a hypothesis strongly suggested by the change in AChE staining) or the medial septal area paced MEC $\theta$ via a relay through the hippocampus.

We reasoned that the most likely relay to achieve the required temporal precision from MSA to MEC would be through the ventral (or temporal) third of HPC, the only portion of HPC which has a monosynaptic projection to EC (Hjorth-Simonsen, 1971; Swanson and Cowan, 1977). Removal of the ventral third of HPC followed by assessments of slow waves and AChE staining in MEC would eliminate this possibility. Such a lesion would be technically difficult and would pose problems of interpretation; it would have to spare any direct fibers from the MSA to the MEC and also not interfere with slow wave recording from the implanted electrodes. Therefore, an altcrnative approach was taken by removing the fimbria, thus disconnecting the MSA from the ventral HPC. If MSA paced MEC $\theta$ rhythm via a relay in the ventral

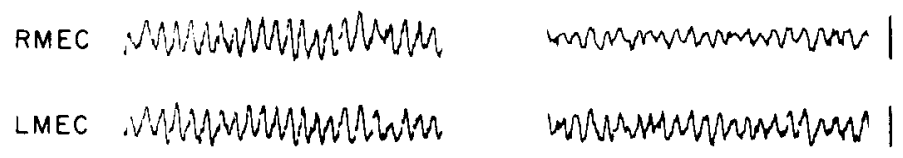

Figure 8. Slow waves from the right DHPC $(R D H P C)$ and the right and left MEC ( $R M E C$ and $L M E C$ ) before and 1 day after a right unilateral dorsal fornix lesion. Calibration bars, $500 \mu \mathrm{V}$ and 1 sec. Data are from rat E28.

HPC or via a direct pathway in the fimbria, this lesion should abolish MEC $\theta$ and deplete AChE ipsilaterally. Such was not the case, however. Unilateral fimbria lesions which did not damage MSA produced only very slight losses of $\theta$ rhythm and slight, if any, losses of AChE staining in ipsilateral MEC. Therefore, MSA cannot pace MEC $\theta$ rhythm via this particular relay through HPC nor does MSA pace MEC directly through fibers carried in the fimbria.

The only possibilities which remained were that MSA paced MEC $\theta$ rhythm via a multisynaptic route through the dorsal HPC or directly via the dorsal fornix. To distinguish between these alternatives, a radiofrequency lesion was placed in the dorsal fornix at the level of the hippocampal flexure. Such a lesion should spare the fibers from MSA to the dorsal HPC, thus sparing a multisynaptic route to MEC, but transect any fibers destined directly for retrohippocampal areas.

In one rat with a unilateral lesion of the dorsal fornix, this dissociation was found. The $\theta$ rhythm in the DHPC was essentially intact, while that in the MEC showed a 47\% loss. This substantive MEC loss is the expected result if MSA paces MEC $\theta$ rhythm via fibers carried in the dorsal fornix. Lesions in two other rats produced approximately equivalent $\theta$ rhythm disruption in DHPC and MEC. These results are expected if the lesion of the dorsal fornix is rostral enough with respect to the DHPC recording electrodes so that fibers destined for DHPC are damaged as well as fibers destined for retrohippocampal areas. In two remaining rats, the attempted dorsal fornix lesions produced substantial disruption of DHPC $\theta$ rhythm and only slight loss of MEC $\theta$ rhythm. This might be the expected finding if the lesion were rostral to the DHPC recording electrodes and were placed in the mediolateral plane so as to spare fibers destined for more caudal sites while transecting fibers destined for the HPC. Thus, none of these data are inconsistent with the hypothesis that a monosynaptic projection from MSA to MEC via the dorsal fornix paces MEC $\theta$ rhythm. However, the data from the two rats which had a greater loss of $\theta$ rhythm in HPC than in MEC are inconsistent with the hypothesis that MEC $\theta$ is paced via a multisynaptic relay in DHPC.

In all of the rats with dorsal fornix lesions, $\theta$ rhythm recovered several months after the lesions were made. Careful examination of the lesions revealed that they all 

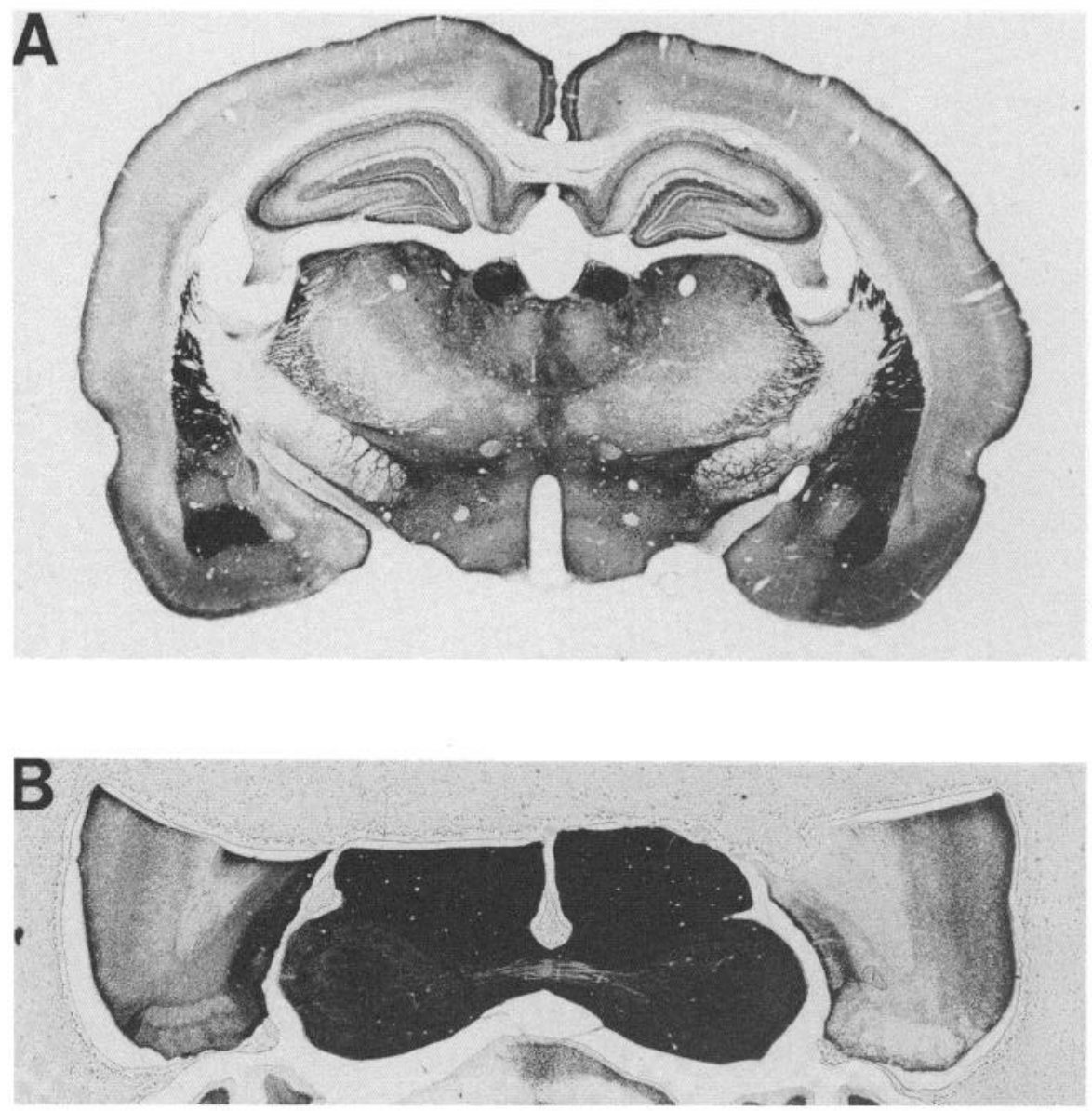

Figure 9. AChE staining pattern in the brain of a rat with a right unilateral suction lesion of dorsal fornix. $A$, A coronal section taken rostral to the lesion site and showing DHPC; $B$, a horizontal section showing MEC. These sections are from rat UDF4.

spared some dorsal fornix fibers. We expect that the early disruption of MEC $\theta$ activity resulted from post-lesion trauma to MSA fibers destined for MEC, not permanent damage to them, or recovery was due to reinnervation of MEC by sprouted MSA fibers which followed the undamaged dorsal fornix fibers to their EC destination. As would be expected in either case, AChE staining in MEC showed little, if any, loss.

Because it is technically extremely difficult to produce a complete lesion of the dorsal fornix in rats which have implanted recording electrodes, in four additional rats without recording electrodes, complete unilateral suction lesions of this bundle were made. The expected dissociation in AChE was found after these lesions: AChE activity in the DHPC was unaltered, while that in ipsilateral MEC was abolished. These results, in conjunction with the high correlation between the presence of $\mathrm{AChE}$ stain and $\theta$ activity, suggest that the pacemaking role of the MSA has been extended to include the MEC $\theta$ rhythm .

In addition, our data revealed that, following fimbria lesions, the ipsilateral ventral LEC suffered a major AChE depletion. Following dorsal fornix suction lesions, AChE staining remained intact in LEC in both dorsal and ventral regions.
These results suggest a topographic organization of the MSA projection to EC which is quite similar to that of the MSA hippocampal projection. As shown schematically in Figure 10, the MSA cholinergic projection which mediates $\theta$ rhythm in DHPC and MEC is carried by the dorsal fornix and the MSA cholinergic projection which mediates $\theta$ rhythm in VHPC is carried by the fimbria.

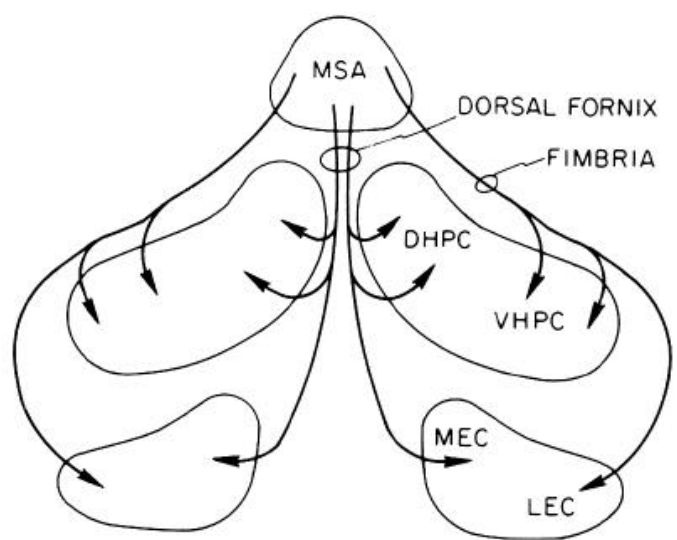

Figure 10. A schematic diagram showing the MSA projections to HPC and EC. See the text for details. 
The fimbria also carries a cholinergic projection to LEC, but there are no data about slow wave activity there.

These results suggest that any modulatory effect that the MSA may have on hippocampal cells also may be present on entorhinal cortical cells. In fact, the location of the $\theta$ rhythm generator or generators in MEC suggests that the MSA may modulate those EC cells which project to the hippocampus.

The second objective of the present study was to determine whether the $\theta$ rhythm and AChE normally present in HPC and EC are necessary for rats to learn and perform a task which is a sensitive measure of hippocampal formation dysfunction. Following destruction of the MSA, rats were impaired in the acquisition of the radial arm maze task, but six of the seven rats did reach criterion performance within 30 test sessions. The magnitude of the behavioral impairment was correlated positively with the loss of $\theta$ and AChE activity in the hippocampus. Although the correlation did not achieve statistical significance in the present experiment, this failure probably reflects the small sample size rather than the absence of a correlation. The correlation was high, and the rank orders for the $\theta$ and $\mathrm{AChE}$ activity loss and the behavioral impairment were identical with the exception of a single point. Also, the addition of any control rat, which would have provided an end point for normal behavior and no $\theta$ or AChE loss, would have provided a statistically significant result even with this small sample size. Thus, a larger sample, and one in which the amount of damage to the MSA varied through a wider range, undoubtedly would have produced a statistically significant result.

The slight behavioral impairment seen in all rats with unilateral fimbria lesions was unexpected. Unilateral radiofrequency fornix-fimbria lesions or EC lesions produced no impairment in this task in previous studies (Olton et al., 1981). Since we observed extensive tissue movement following the suction lesion, we suspect that the behavioral deficit may have been due to nonspecific traumatic brain damage. Without appropriate control lesions, we are unable to distinguish between this possibility and a geniune effect due to our selective fimbria lesion.

In conclusion, we believe that disruptions of the $\theta$ rhythm system result in a severe impairment in acquisition of the radial arm maze task but do not prevent the rats from ultimately learning the task. These results are in agreement with those of Winson (1978), who also measured performance of rats on a spatial memory task following lesions of the medial septal nucleus which eliminated $\theta$ rhythm. In his circular maze task, cues were present to indicate the correct reward location during the early training period. Despite this presence of cues, medial septal nucleus-lesioned rats learned the task slower than normal animals or operated controls. These results are quite markedly different from the results seen following bilateral fornix-fimbria or EC lesions where rats perform at chance levels on the radial arm maze throughout extensive post-lesion test periods (Olton et al., 1978, 1979,1981 ). Our data suggest either that working memory for this spatial task remains intact following MSA lesions or that any small bits of MSA left intact may be or may become adequate to mediate a memory function.

\section{References}

Apostol, G., and O. D. Creutzfeldt (1974) Crosscorrelation between the activity of septal units and hippocampal EEG during arousal. Brain Res. 67: 65-75.

Artemenko, D. P. (1972) Role of hippocampal neurons in thetawave generation. Nierofiziologiya 4: 531-539.

Becker, J. T., J. A. Walker, and D. S. Olton (1980) Neuroanatomical bases of spatial memory. Brain Res. 200: 307-320.

Bland, B. H., and I. Q. Whishaw (1976) Generators and topography of hippocampal theta (RSA) in the anesthetized and freely moving rat. Brain Res. 118: 259-280.

Bland, B. H., P. Andersen, and T. Ganes (1975) Two generators of hippocampal theta activity in rabbits. Brain Res. 94: 199218.

Fox, S. E. (1980) Hippocampal pyramidal cells depolarize on the negative phase of dentate theta rhythm in urethane anesthetized rats. Soc. Neurosci. Abstr. 6: 564 .

Fujita, Y., and T. Sato (1964) Intracellular records from hippocampal pyramidal cells in rabbit during theta rhythm activity. J. Neurophysiol. 27: 1011-1025.

Gogolák, G., H. Petsche, J. Sterc, and C. Stumpf (1967) Septum cell activity in the rabbit under reticular stimulation. Brain Res. 5: 508-510.

Gogolák, G., C. Stumpf, H. Petsche, and J. Sterc (1968) The firing pattern of septal neurons and the form of the hippocampal theta wave. Brain Res. 7: 201-207.

Green, J. D., and H. Petsche (1961) Hippocampal electrical activity. II. Virtual generators. Electroencephalogr. Clin. Neurophysiol. 13: 847-853.

Green, J. D., D. S. Maxwell, W. J. Schindler, and C. Stumpf (1960) Rabbit EEG 'theta' rhythm: Its anatomical source and relation to activity in single neurons. J. Neurophysiol. 23: 403-420.

Green, K. F., and J. N. P. Rawlins (1979) Hippocampal theta in rats under urethane: Generators and phase relations. Electroencephalogr. Clin. Neurophysiol. 47: 420-429.

Hjorth-Simonsen, A. (1971) Hippocampal efferents to the ipsilateral entorhinal area: An experimental study in the rat. J. Comp. Neurol. 142: 417-438.

Honig, W. K. (1978) Studies of working memory in the pigeon. In Cognitive Processes in Animal Behavior, S. H. Hulse, H. Fowler, and W. K. Honig, eds., pp. 211-248, Erlbaum, Hillsdale, NJ.

Humason, G. L. (1979) Animal Tissue Techniques, pp. 191194, W. H. Freeman and Co., San Francisco.

McLennan, H., and J. J. Miller (1974) The hippocampal control of neuronal discharges in the septum of the rat. J. Physiol. (Lond.) 237: 607-624.

McLennan, H., and J. J. Miller (1976) Frequency-related inhibitory mechanisms controlling rhythmical activity in the septal area. J. Physiol. (Lond.) 254: 827-841.

Meibach, R. C., and A. Siegel (1977) Efferent connections of the septal area in the rat: An analysis utilizing retrograde and anterograde transport methods. Brain Res. 119: 1-20.

Mellgren, S. I., and B. Srebro (1973) Changes in acetylcholinesterase and distribution of degenerating fibres in the hippocampal region after septal lesions in the rat. Brain Res. 52: 19-36.

Mitchell, S. J., and J. B. Ranck, Jr. (1980) Generation of theta rhythm in medial entorhinal cortex of freely-moving rats. Brain Res. 189: 49-66.

Naik, N. T. (1963) Technical variations in Koelle's histochemical method for demonstrating cholinesterase activity. Q. J. Microsc. Sci. 104: 89-100. 
Olton, D. S., and R. J. Samuelson (1976) Remembrance of places passed. Spatial memory in rats. J. Exp. Psychol. (Anim. Behav. Proc.) 2: 97-116.

Olton, D. S., J. A. Walker, and F. H. Gage (1978) Hippocampal connections and spatial discrimination. Brain Res. 139: 295308.

Olton, D. S., J. T. Becker, and G. E. Handelmann (1979) Hippocampus, space, and memory. Behav. Brain Sci. 2: 313365.

Olton, D. S., J. A. Walker, and W. A. Wolf (1981) A disconnection analysis of hippocampal function. Brain Res., in press.

Petsche, H., C. Stumpf, and G. Gogolák (1962) The significance of the rabbit's septum as a relay station between the midbrain and the hippocampus. I. The control of hippocampal arousal activity by the septum cells. Electroencephalogr. Clin. Neurophysiol. 14: 202-211.

Petsche, H., G. Gogolák, and P. A. van Zwieten (1965) Rhythmicity of septal cell discharges at various levels of reticular excitation. Electroencephalogr. Clin. Neurophysiol. 19: 2533.

Raisman, G. (1966) The connexions of the septum. Brain 89: 317-348.

Ramón y Cajal, S. (1955) Studies on the Cerebral Cortex (Limbic Structures), L. M. Kraft, translator, London, LloydLuke Ltd, Year Book Medical Publishers, Chicago.

Ranck, J. B., Jr. (1976) Behavioral correlates and firing repertoires of neurons in septal nuclei in unrestrained rats. In The Septal Nuclei, J. F. DeFrance, ed., pp. 423-462, Plenum Press, New York.
Rawlins, J. N. P., J. Feldon, and J. A. Gray (1979) Septohippocampal connections and the hippocampal theta rhythm. Exp. Brain Res. 37: 49-63.

Segal, M. (1977) Afferents to the entorhinal cortex of the rat studied by the method of retrograde transport of horseradish peroxidase. Exp. Neurol. 57: 750-765.

Siegel, A., and J. P. Tassoni (1971) Differential efferent projections of the lateral and medial septal nuclei to the hippocampus in the cat. Brain Behav. Evol. 4: 201-219.

Storm-Mathisen, J., and T. W. Blackstad (1964) Cholinesterase in the hippocampal region. Acta Anat. (Basel) 56: 216-253.

Swanson, L. W., and W. M. Cowan (1977) An autoradiographic study of the organization of the efferent connections of the hippocampal formation in the rat. J. Comp. Neurol. 172: 4984

Wilson, C. L., B. C. Motter, and D. B. Lindsley (1976) Influences of hypothalamic stimulation upon septal and hippocampal electrical activity in the cat. Brain Res. 197: 55-68.

Winson, J. (1974) Patterns of hippocampal theta rhythm in the freely-moving rat. Electroencephalogr. Clin. Neurophysiol. 36: 291-301.

Winson, J. (1976a) Hippocampal theta rhythm. I. Depth profiles in the curarized rat. Brain Res. 103: 57-70.

Winson, J. (1976b) Hippocampal theta rhythm. II. Depth profiles in the freely moving rabbit. Brain Res. 103: 71-79.

Winson, J. (1978) Loss of hippocampal theta rhythm results in spatial memory deficit in the rat. Science 201: 160-163. 